\title{
An estimate of rate of deviation from NCCN guideline recommendations for central nervous system imaging in trials forming basis for drug approval in first line advanced non-small cell lung cancer (NSCLC)
}

John Sharp ${ }^{1}$ and Vinay Prasad $2,3^{*}$

\begin{abstract}
Importance: It is unknown whether and to what degree trials submitted to the US FDA to support drug approval adhere to NCCN guideline-recommended care in their baseline and surveillance CNS imaging protocols.

Objective: We sought to characterize the frequency with which the trials cited in US FDA drug approvals for first line advanced NSCLC between 2015 and 2020 deviated from NCCN guideline-recommended care for baseline and surveillance CNS imaging.

Design, setting, and participants: Retrospective observational analysis using publicly available data of (1) list of trials cited by the FDA in drug approvals for first line advanced NSCLC from 2015 to 2020 (2) individual trial protocols (3) published trial data and supplementary appendices (4) archived versions of the NCCN guidelines for NSCLC from 2009 to 2018 (the years during which the trials were enrolling).

Main outcomes and measures: Estimated percentage of trials for first line advanced NSCLC leading to FDA approval which deviated from NCCN guideline-recommended care with regards to CNS baseline and surveillance imaging.

Results: A total of 14 studies that had been cited in FDA drug approvals for first line advanced NSCLC met our inclusion criteria between January 1, 2015 and September 30, 2020. Of these trials, 8 (57.1\%) deviated from NCCN guidelines in their baseline CNS imaging requirement. The frequency of re-assessment of CNS disease was variable amongst trials as well, with 9 (64.3\%) deviating from NCCN recommendations.

Conclusions and relevance: The trials supporting US FDA drug approvals in first line advanced NSCLC often have CNS imaging requirements that do not adhere to NCCN guidelines. Many trials permit alternative, substandard methods and the proportion of patients undergoing each modality is uniformly not reported. Nonstandard CNS surveillance protocols are common. To best serve patients with advanced NSCLC in the US, drug approvals by the FDA must be based on trials that mirror clinical practice and have imaging requirements consistent with current US standard of care.
\end{abstract}

*Correspondence: Vinayak.prasad@ucsf.edu

2 Department of Medicine, University of California San Francisco, 550 16th

St., San Francisco, California CA 94158, USA

Full list of author information is available at the end of the article original author(s) and the source, provide a link to the Creative Commons licence, and indicate if changes were made. The images or other third party material in this article are included in the article's Creative Commons licence, unless indicated otherwise in a credit line to the material. If material is not included in the article's Creative Commons licence and your intended use is not permitted by statutory regulation or exceeds the permitted use, you will need to obtain permission directly from the copyright holder. To view a copy of this licence, visit http://creativecommons.org/licenses/by/4.0/. The Creative Commons Public Domain Dedication waiver (http://creativeco mmons.org/publicdomain/zero/1.0/) applies to the data made available in this article, unless otherwise stated in a credit line to the data. 


\section{Key points}

Question How many drugs for first line advanced nonsmall cell lung cancer (NSCLC) received US Food and Drug Administration (FDA) approval on the basis of trials that deviated from National Comprehensive Cancer Network (NCCN) guideline recommendations regarding baseline and surveillance central nervous system (CNS) imaging?

Findings In this retrospective observational study among 14 trials for drugs used in first line advanced NSCLC that formed the basis for FDA approval between 2015 and 2020, 8 (57.1\%) deviated from NCCN guidelinerecommended care for baseline CNS imaging. 9 (64.3\%) trials had CNS surveillance imaging protocols that deviated from NCCN guideline-recommended care.

Meaning Many of the trials that led to US FDA approval of drugs in first line advanced NSCLC relied on baseline and surveillance CNS imaging protocols that deviated from NCCN guideline-recommended care. When discrepancies in baseline and surveillance CNS imaging exist between clinical trial protocols and real world practice, the generalizability of benefits observed in the trial population becomes less certain.

\section{Introduction}

Over 228,000 people will be diagnosed with lung cancer each year in the United States and the vast majority will present with non-small cell lung cancer (NSCLC) [1] . Lung cancer is the leading cause of cancer-related mortality in the United States and 5-year survival rates remain below 25\%. A 1995 BMJ meta-analysis of 8 clinical trials comparing platinum-based chemotherapy to best supportive care demonstrated the most effective chemotherapy regimens of the day increased the one-year survival rate from 5 to $15 \%$ [2]. New therapeutic options have improved patient outcomes, with the average one-year survival for advanced forms of the disease now exceeding 25\% [1] . Continued improvement in patient outcomes relies upon well-designed clinical trials comparing novel therapeutic agents to the current best standard of care. Unfortunately, trials are not always designed in this fashion. Many trials in oncology utilize control arms that are disputed and potentially inferior to other available options $[3,4]$. A reliance on surrogate endpoints and the inappropriate use of post-protocol therapies (or crossover) have also been cited as deficiencies in pivotal registration studies submitted to the US FDA [5] .

However, what remains unknown is whether imaging at baseline and to monitor disease - in these studies mirrors US standards of care. Inadequate staging at baseline, particularly of the CNS, may result in occult intracranial disease going untreated, which has both prognostic and therapeutic implications. Frequent follow up imaging of the CNS may artificially demonstrate gains in progression, though these may not be recapitulated in clinical settings where frequent surveillance CNS imaging is nonstandard. For these reasons, we sought to investigate the use of CNS imaging in clinical trials of drugs approved by the US FDA to treat advanced NSCLC and how this differs from the standard of care in US clinical practice. The National Comprehensive Cancer Network (NCCN) guidelines, derived from clinical trial data and expert consensus, are used by as many as $95 \%$ of US oncologists [6] and are now Medicare compendia, which mandates that Medicare base payment decisions on them and thus codifies these guidelines as a US standard bearer.

\section{Methods \\ Overview}

Our study was not submitted for institutional review board approval because it did not involve healthcare records and all data are publicly available. The study was conducted between September 23, 2020 to December 8, 2020. We sought to estimate the percentage of clinical trials leading to FDA drug approval for first line advanced NSCLC that had protocols which deviated from NCCN guideline recommendations, with regards to CNS baseline and surveillance imaging. We examined all FDA approvals for first line advanced NSCLC between January 1, 2015 and September 23, 2020. We then considered only phase 3 or combined phase $2 / 3$ trials that were cited in FDA announcements as the basis for drug approvals in the first line advanced NSCLC space. We analyzed only those studies with readily available protocol documents to determine the nature of the baseline and surveillance CNS imaging within the trial. We compared these protocols to the archived versions of NCCN guidelines for advanced NSCLC that would have been relevant during enrollment of these trials. We report on the findings of the protocols' deviation from NCCN guidelines.

\section{Data set \\ Study selection}

We examined all US FDA oncology drug approvals for first line advanced NSCLC from January 1, 2015 through September 23, 2020 based on data available at the FDA website. We included phase 3 and combined phase $2 / 3$ trials with a standard-of-care comparator arm that had publicly available complete protocol documents. We retrieved the published manuscripts of the clinical trials cited as the basis for each approval as well as their supplementary appendices and protocols. We did not consider trials that had not yet been published at the time of investigation (CHECKMATE 9LA). We also did not consider phase 1 , phase 2 , latter line phase 3 trials or accelerated approvals. 


\section{Data extracted}

For each cited trial, we catalogued the dates of active enrollment of subjects, stages of subjects enrolled, name of the drug/combination therapy receiving approval on the basis of the trial, comparator treatment arm, date of approval, specific treatment indication, whether the drug was a new molecular entity, whether the trial was multinational, the continents on which the trial was conducted, whether brain MRI was required at baseline, alternative accepted CNS imaging modalities within the trial, and the schedule of assessment of CNS disease. Additionally, in cases where brain MRI was not mandated for CNS staging in all subjects, we examined data reported in the trial manuscripts and supplements for information on the frequency with which alternative CNS assessments were performed. Archived versions of the NCCN guidelines for NSCLC were reviewed for their recommendations regarding baseline and surveillance CNS imaging and these were compared to the protocols of the trials considered.

\section{Determining NCCN guideline recommendations}

Archived versions of the NCCN guidelines for NSCLC were obtained with the express consent of the NCCN. These guidelines had been published from November 30, 2009 to August 17, 2018, which encompassed the entire enrollment periods of all trials under consideration. As many versions of these guidelines are published each year, each individual version of the guidelines was reviewed for its recommendations.

To determine the recommendations for baseline CNS imaging, the Evaluation and Treatment sections of the NCCN guidelines for stages IIIA, IIIB, and IV were reviewed. Because these trials only considered advanced NSCLC, the recommendations for stages less than IIIA were not reviewed. Each version of the guidelines was categorized by its recommendation of baseline brain MRI as being either 1) indicated or 2) not indicated. The recommendations from the versions of the NCCN guidelines encompassing each trial's enrollment period were then used to compare the contemporaneous NCCN guideline-recommended care to each trial protocol.

To determine the recommendations for surveillance CNS imaging, the Surveillance section of the NCCN guidelines were reviewed. The surveillance recommendations for all stages of NSCLC, ranging from no evidence of disease to stage IV were reviewed to encompass the recommended reassessments for all potential degree of response subgroups within each trial. For each version of the NCCN guidelines, the surveillance recommendation was categorized as 1) brain MRI routinely indicated or 2) brain MRI not routinely indicated. The recommendations from the versions of the NCCN guidelines encompassing each trial's enrollment period were then used to compare the contemporaneous NCCN guideline-recommended care to each trial protocol.

\section{Determining baseline CNS imaging requirements}

To determine the number of trial protocols deviating from NCCN guidelines for baseline CNS imaging, all trial protocols were reviewed for their baseline CNS imaging requirements. Methods sections, trial flow charts, and schedule of assessments tables were reviewed to determine how CNS imaging was performed at baseline. Protocols were then compared to contemporaneous NCCN guidelines and categorized as either 1) adhering to NCCN guidelines for CNS imaging at baseline for all subjects, 2) not adhering to NCCN guidelines for CNS imaging at baseline for all subjects or 3) unclear. For protocols falling into the second category, a text description of CNS imaging requirements was collected. We then divided the number of trials that deviated from NCCN guidelines for baseline CNS imaging by the total number of trials considered to determine the percentage of trials not meeting NCCN guideline recommendations for CNS imaging.

\section{Determining reporting of CNS imaging modality}

All protocols were reviewed for CNS imaging requirements as described above. For protocols that did not adhere to NCCN guidelines for baseline CNS imaging for all subjects, text descriptions of alternative baseline CNS imaging modalities and practices, as described in the Methods sections, were recorded. The published trials and their supplementary appendices were then reviewed for reporting on the frequency with which alternative CNS imaging modalities and practices were performed. Where available, these values were recorded.

\section{Determining CNS surveillance imaging requirements}

The methods sections, trial flow charts, and schedule of assessments tables of all trial protocols were reviewed to determine the frequency with which CNS reassessment was required. The protocols were categorized as 1) mandating CNS reassessment with each scheduled assessment of disease for all subjects, 2) mandating CNS reassessment with each scheduled assessment of disease for subjects with known baseline CNS metastatic disease, 3) CNS reassessment as clinically indicated or 4) unclear. We calculated the percentage of trials within each category by dividing the number of trials within each category by the total number of trials considered.

We further compared each trial protocol's schedule for CNS reassessment to the schedule for CNS reassessment 


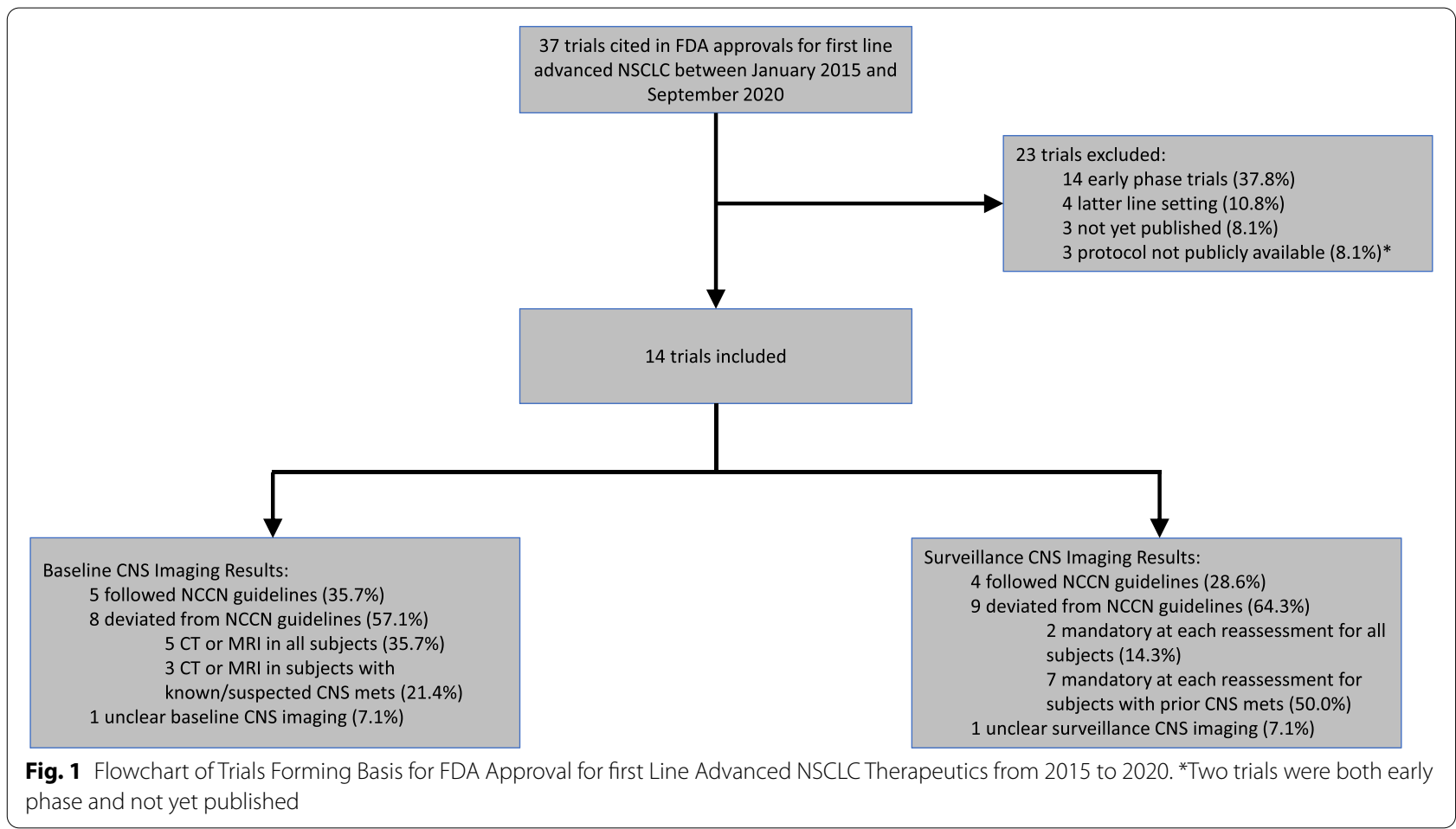

recommended by the NCCN guidelines for the dates corresponding to the enrollment period for each individual trial. Because the NCCN recommendation for CNS reassessment did not vary by burden of disease (i.e no evidence of disease vs stage IV), subgroup analysis by treatment response was not performed. We then calculated the percentage of trials deviating from $\mathrm{NCCN}$ guideline recommended CNS surveillance imaging by dividing the number of trials with protocols that did not adhere to the contemporaneous NCCN guidelines by the total number of trials considered.

\section{Statistical analysis}

We sought to provide a descriptive estimate of the percentage of trials leading to FDA drug approval for advanced NSCLC that deviated from NCCN guidelinerecommended care for baseline and surveillance CNS imaging. Analysis was performed using Microsoft Excel.

\section{Results}

We examined 37 FDA approvals for first-line treatment of advanced NSCLC between January 1, 2015 and September 23, 2020. Of these, 14 (37.8\%) met our inclusion criteria, as seen in Fig. 1 . The main reasons for exclusion were pivotal data emerged from a phase 1 or 2 trial $(n=14)$, latter line setting $(n=4)$, trial was not yet published $(n=3)$, and protocol was not publicly available $(n=3)$. Two trials were both early phase trials and unpublished.

\section{Results of NCCN guideline audit}

Forty versions of the NCCN guidelines were published between November 30, 2009 to August 17, 2018. For stage IIIA, IIIB, and IV NSCLC, the NCCN recommended MRI brain with contrast as baseline CNS imaging in $40(100 \%)$ of the versions. For stage IIIA, IIIB, and IV NSCLC, the NCCN stated that surveillance brain MRI is not routinely indicated in $40(100 \%)$ of the versions.

\section{Baseline CNS imaging assessments}

As seen in Table 1 , of the 14 trials we considered, 8 (57.1\%) deviated from NCCN guidelines with respect to baseline CNS imaging requirements. 5 (35.7\%) allowed either CT or MR but did require baseline CNS imaging in all participants. 3 (21.4\%) mandated some form of baseline CNS imaging, either MRI or CT, in all subjects with a known or suspected history of CNS metastases. Only 5 (35.7\%) explicitly mandated baseline brain MRI in all trial participants as recommended by the NCCN. 1 (7.1\%) did not have explicit baseline CNS imaging requirements elaborated in their protocols.

Of the 9 (64.3\%) studies that did not explicitly mandate NCCN guideline-recommended baseline CNS imaging (including the 1 study with unclear 
Table 1 Summary of trials cited as basis for FDA approval for drugs used in first line advanced NSCLC and their baseline and surveillance CNS imaging requirements

\begin{tabular}{|c|c|c|c|c|}
\hline Trial Name & Drug and Indication & NCT & Baseline CNS Imaging Required & Surveillance CNS Imaging Required \\
\hline RELAY & $\begin{array}{l}\text { Ramucirumab + Erlotinib, first line } \\
\text { EGFR (19del, L858R) }\end{array}$ & NCT02411448 & MRI brain required for all subjects & As clinically indicated \\
\hline ALTA-1 L & Brigatinib, first line ALK mutated & NCT02737501 & MRI brain required for all subjects & $\begin{array}{l}\text { At regular imaging intervals in all } \\
\text { subjects }\end{array}$ \\
\hline Impower110 & Atezolizumab, first line PDL1 high & NCT02409342 & $\begin{array}{l}\mathrm{CT} \text { or MRI brain required for all } \\
\text { subjects }\end{array}$ & $\begin{array}{l}\text { At regular imaging intervals in sub- } \\
\text { jects with CNS disease }\end{array}$ \\
\hline CHECKMATE-227 & $\begin{array}{l}\text { Ipilimumab + Nivolumab, first line } \\
\text { PDL1> } 1 \%\end{array}$ & NCT02477826 & MRI brain required for all subjects & $\begin{array}{l}\text { At regular imaging intervals in sub- } \\
\text { jects with CNS disease }\end{array}$ \\
\hline IMpower130 & $\begin{array}{l}\text { Atezolizumab with carboplatin/ } \\
\text { protein-bound paclitaxel, first line } \\
\text { non-squamous }\end{array}$ & NCT02367781 & $\begin{array}{l}\mathrm{CT} \text { or MRI brain required for all } \\
\text { subjects }\end{array}$ & As clinically indicated \\
\hline KEYNOTE-042 & Pembrolizumab, first line PDL1 > 1\% & NCT02220894 & Unclear* & Unclear* \\
\hline KEYNOTE-407 & $\begin{array}{l}\text { Pembrolizumab + carboplatin/pacli- } \\
\text { taxel, first line }\end{array}$ & NCT02775435 & CT or MRI brain accepted** & $\begin{array}{l}\text { At regular imaging intervals in sub- } \\
\text { jects with CNS disease }\end{array}$ \\
\hline ARCHER 1050 & $\begin{array}{l}\text { Dacomitinib, first line EGFR (19del, } \\
\text { L858R) }\end{array}$ & NCT01774721 & $\begin{array}{l}\mathrm{CT} \text { or MRI brain required for all } \\
\text { subjects }\end{array}$ & As clinically indicated \\
\hline KEYNOTE-189 & $\begin{array}{l}\text { Pembrolizumab + pemetrexed/car- } \\
\text { boplatin, first line }\end{array}$ & NCT02578680 & CT or MRI brain accepted** & $\begin{array}{l}\text { At regular imaging intervals in sub- } \\
\text { jects with CNS disease }\end{array}$ \\
\hline FLAURA & $\begin{array}{l}\text { Osimertinib, first line EGFR (19del, } \\
\text { L858R) }\end{array}$ & NCT02296125 & $\begin{array}{l}\text { CT or MRI required if known/sus- } \\
\text { pected CNS disease }\end{array}$ & $\begin{array}{l}\text { At regular imaging intervals in sub- } \\
\text { jects with CNS disease }\end{array}$ \\
\hline ASCEND-4 & Ceritinib, first line ALK mutated & NCT01828099 & $\begin{array}{l}\text { CT or MRI brain required for all } \\
\text { subjects }\end{array}$ & $\begin{array}{l}\text { At regular imaging intervals in sub- } \\
\text { jects with CNS disease }\end{array}$ \\
\hline ALEX & Alectinib, first line ALK mutated & NCT02075840 & MRI brain required for all subjects & $\begin{array}{l}\text { At regular imaging intervals in all } \\
\text { subjects }\end{array}$ \\
\hline KEYNOTE-024 & Pembrolizumab, first line PDL1 high & NCT02142738 & MRI brain required for all subjects & As clinically indicated \\
\hline SQUIRE & $\begin{array}{l}\text { Necitumumab + gemcitabine/cispl- } \\
\text { atin, first line squamous }\end{array}$ & NCT00981058 & $\begin{array}{l}\text { CT or MRI brain required for all } \\
\text { subjects }\end{array}$ & $\begin{array}{l}\text { At regular imaging intervals in sub- } \\
\text { jects with CNS disease }\end{array}$ \\
\hline
\end{tabular}

*Protocol, supplement, and manuscript do not explicitly discuss requirement

**Protocol is not explicit if all subjects or only subjects with history of CNS disease underwent baseline CNS screening

requirements), $0(0 \%)$ reported the frequency with which alternative modalities, such as $\mathrm{CT}$ brain, were used.

\section{CNS surveillance strategies}

Of the 14 trials considered, 9 (64.3\%) deviated from NCCN recommended CNS surveillance strategies. 2 (14.3\%) mandated CNS imaging at each scheduled disease assessment in all patients. 7 (50.0\%) mandated CNS imaging at each scheduled disease assessment in all patients with a known history of CNS disease at baseline. Four trials $(28.6 \%)$ specified that repeat CNS assessment should occur as clinically indicated, consistent with NCCN guidelines. 1 (7.1\%) had unclear CNS surveillance protocols.

\section{Multinational trials}

All $14(100 \%)$ trials that were examined occurred in the multinational setting. 2 (14.3\%) trials enrolled no patients in the United States at all.

\section{New molecular entities}

The 14 trials considered evaluated 9 different drugs. Of the 9 drugs, 3 (33.3\%) were new molecular entities.

\section{Discussion}

Clinical trials supporting US Food and Drug Administration approval for advanced NSCLC therapies should ideally assess efficacy of novel therapies over available standard of care in the United States. When the care provided in a trial setting deviates significantly from clinical practice, the conclusions of the trial are not as generalizable to real world patients. Prior analyses have examined deviations from standard of care in the form of deficiencies in control arm choice and post-protocol therapies; however, our study is the first to examine the role of intracranial imaging [3-5] .

We find that $57.1 \%$ of trials do not adhere to NCCN guideline recommendations for baseline CNS imaging, either by accepting CT brain, which is less sensitive than the US standard of MRI, or by failing to screen all subjects for CNS disease, or both. 35.7\% performed baseline 
CNS imaging adherent to NCCN guidelines. $7.1 \%$ had unclear baseline CNS imaging requirements. The frequency with which substandard imaging was employed was universally not reported. After initiation of therapy, $64.3 \%$ performed surveillance CNS imaging in a manner discordant with NCCN guideline recommendations, $28.6 \%$ performed CNS imaging as appropriately indicated, and $7.1 \%$ had unclear CNS surveillance imaging protocols.

To our knowledge, this is the first investigation to report on baseline and surveillance imaging protocols in trials leading to drug approvals in first line advanced NSCLC. The findings raise important questions regarding both the care provided to patients within the trials as well as the generalizability of the benefits reported in the trial setting to patients with advanced NSCLC receiving treatment in the US.

First, deviations from NCCN guidelines regarding baseline CNS imaging have the potential to "downstage" patients at baseline. MRI has been the standard NCCN recommended imaging modality to assess for CNS disease since at least 2009, the earliest any of the trials considered began enrollment. This is due to its superior sensitivity compared to CT [7, 8], particularly for metastases less than $5 \mathrm{~mm}$ in size, in addition to its lack of ionizing radiation and superior tissue resolution. By accepting CT brain studies in place of MRI brain with contrast, as is recommended by the NCCN in the workup of all NSCLC patients with advanced disease (i.e. stages IIIA and above), CNS metastases present at baseline, particularly small ones, may be missed initially but subsequently scored as progression of disease when detected on surveillance imaging. If a trial is assessing an adjuvant therapy, lack of appropriate brain imaging may miss occult metastatic disease. Substandard baseline CNS imaging raises important ethical questions as it would be beneath the US standard of care to deprive patients with CNS disease appropriate radiation therapy. Additionally, significant differences with respect to prognosis have been described [9] between patients with CNS metastases at baseline and those without. In trials accepting alternative CNS imaging modalities, imbalances in the proportion of patients undergoing baseline brain MRI between treatment arms could contribute to differences in outcomes related to presence of occult CNS disease rather than the effect of the therapeutic intervention. It is difficult to interpret how much of an impact these practices have on the overall results of many of these trials as none report the proportion of subjects undergoing each modality of baseline CNS screening.

The other deviation from NCCN guideline-recommended care we observed is that many trials only required baseline CNS imaging in patients with a known or suspected history of CNS disease. While it is possible that diagnostic imaging consistent with the local standard of care was obtained prior to trial enrollment for these patients, it cannot be assumed that local standard of care was consistent with the US standard of care due to the widely multinational nature of these trials. Brain metastases are estimated to occur in $41 \%$ of patients with NSCLC [10] and by accepting head CT in place of the diagnostically superior brain MRI, which is the standard of care in the US, it is highly plausible that a proportion of patients in these trials had undetected baseline CNS disease. Because the trials do not report the proportion of patients in each arm undergoing each CNS imaging modality, the effect on the results is uncertain. Regardless, one way to be more confident that clinical benefits observed in trials will translate to patients in US oncology clinics is for the FDA to ensure that trials that form the basis for drug approval have protocols that adhere to US standard of care with regards to their imaging.

Second, deviations in CNS surveillance imaging from NCCN guidelines create confusion regarding a drug's benefit. This is particularly relevant in the case of novel agents targeting the ALK pathway (alectinib, brigatinib), which have been touted specifically for their superior CNS penetration. By mandating CNS reassessments at each scheduled imaging interval, as was done in trials investigating these agents, a perceived benefit of better disease control within the CNS was observed. Indeed, a lower cumulative incidence of CNS progression and a superior CNS objective response rate, respectively, were reported as secondary outcomes in these trial manuscripts $[11,12]$. However, this is not what is routinely done in real world Oncology clinics. It remains unclear whether this radiologic benefit observed in the setting of serial asymptomatic imaging is necessarily one that translates into a clinical benefit when imaging occurs in response to symptoms, as it does in NCCN guidelinedirected care.

Third, all trials we examined occurred in the multinational setting, with two trials enrolling no patients at all in the United States, yet they have formed the basis for approval by the FDA for use in the United States [13-24]. While this is not inherently problematic, as well done randomized controlled trials can be performed in many countries, if the care provided in the trial is below the standard of care in the United States, this creates problems with the generalizability of any observed benefit to patients in the United States. It may be that accepting alternate CNS imaging modalities such as $\mathrm{CT}$ is a practical necessity based on local availability, but this is not acceptable care for patients with advanced NSCLC in the US. Imbalances in the proportion of patients undergoing brain MRI between treatment arms could represent 
a potential confounder as patients with undetected CNS disease have an overall worse prognosis a priori compared to those without CNS disease. Therefore, the FDA must interpret trials which implement alternative means of CNS disease assessment with the utmost caution.

\section{Limitations}

A number of limitations to our study must be acknowledged. First, this study looked specifically at the space of advanced NSCLC. The particular pattern of deviation from US standards of care may be unique to this space.

Second, as we only examined trials with publicly available protocols and supplemental appendices, it is possible that we overestimate the number of trials deviating from current US standard of care. We excluded three trials due to their protocols not being readily accessible and designated two included trials as having "unclear" imaging and surveillance protocols and it is possible that the practices in these trials was entirely consistent with US standard practices.

Third, we only examined drugs granted approval after phase III trials in which comparison to a control group is undertaken. As many drugs received accelerated approval on the basis of phase I and II trials, it is possible that the protocols of these trials adhered to US standards with regard to baseline and surveillance imaging. We excluded 14 trials due to their phase I or II nature.

Fourth, it must be stated that it is possible, even likely, that the data regarding the frequency of alternate baseline CNS imaging modality use exists. We did not submit requests for additional data from the trials in which either baseline CT or MRI were permissible. For the purposes of this investigation, the fact that these data are not publicly available is what renders these trials difficult to completely interpret. When alternate modes of CNS imaging besides that recommended by the most up to date guidelines are used, it should be reported in the trial, or at least in the supplementary materials.

\section{Conclusion}

While great strides forward have been made with regard to therapeutics for advanced NSCLC, continued progress depends upon well-designed clinical trials that rigorously test novel agents against existing standards of care. Trial protocols that fall below the current US standard of care have the potential to create confusion about a new therapeutic agent's true benefit by "downstaging" subjects at baseline, missing known metastatic disease, introducing confounders if imbalances exist in imaging techniques between treatment arms, as well as detecting asymptomatic CNS progression that might not have been detected in real world settings. Additionally, it raises ethical concerns about the adequacy of care provided for patients within the trials as CNS disease may be going undetected and undertreated. Nevertheless, these trials are cited as the basis for new drug approvals by the FDA. We found that $57.1 \%$ of trials in first line advanced NSCLC did not strictly adhere to NCCN recommended baseline CNS imaging and that, when alternatives were used, the alternate modality was not reported. Additionally, $64.3 \%$ of trials were not strictly adherent to NCCN recommendations for CNS surveillance strategies. These somewhat subtle deviations from current US standard of care for advanced NSCLC have the potential to create confusion regarding a drug's true benefit. In order to better serve patients with advanced NSCLC in the US, trials which form the basis for drug approvals by the FDA must adhere to current best practices which involves adherence to standard CNS baseline imaging and surveillance protocols.

\section{Abbreviations}

NSCLC: Non-small cell lung cancer; FDA: Food and Drug Administration; NCCN: National Comprehensive Cancer Network; CNS: Central Nervous System; CT: Computerized Tomography; MRI: Magnetic Resonance Imaging.

\section{Acknowledgements}

N/A

\section{Authors' contributions}

John Sharp: Data curation, Investigation, Formal analysis, Visualization, Writing - original draft, Writing - review \& editing. Vinay Prasad: Conceptualization, Methodology, Supervision, Writing - review \& editing. The author(s) read and approved the final manuscript.

\section{Funding}

Vinay Prasad is funded by Arnold Ventures. Grant number N/A.

\section{Availability of data and materials}

The datasets used and/or analysed during the current study are available from the corresponding author on reasonable request.

\section{Declarations}

Ethics approval and consent to participate

Not applicable. This manuscript does not report on or involve the use of any animal or human data or tissue.

Consent for publication

Not applicable.

\section{Competing interests}

Vinay Prasad discloses: (Research funding) Arnold Ventures. (Royalties) Johns Hopkins Press, Medscape, MedPage. (Consulting) UnitedHealthcare. (Speaking fees) Evicore. New Century Health. (Other) Plenary Session podcast has Patreon backers.

\section{Author details}

${ }^{1}$ Department of Medicine, UCLA Health, Los Angeles, California, USA. ${ }^{2}$ Department of Medicine, University of California San Francisco, 550 16th St., San Francisco, California CA 94158, USA. ${ }^{3}$ Department of Epidemiology and Biostatistics, University of California San Francisco, 550 16th St., San Francisco, California CA 94158, USA. 
Received: 17 May 2021 Accepted: 4 January 2022

Published online: 16 January 2022

\section{References}

1. Cancer of the lung and bronchus - cancer stat facts. SEER.

2. Chemotherapy in non-small cell lung cancer: a meta-analysis using updated data on individual patients from 52 randomised clinical trials. Non-small Cell Lung Cancer Collaborative Group. BMJ. 1995:311(7010):899-909.

3. Hilal T, Sonbol MB, Prasad V. Analysis of control arm quality in randomized clinical trials leading to anticancer drug approval by the us food and drug administration. JAMA Oncol. 2019;5(6):887-92.

4. Dear RF, McGeechan K, Barnet MB, Barratt AL, Tattersall MHN. "Standard care" in cancer clinical trials: an analysis of care provided to women in the control arms of breast cancer clinical trials. J Natl Compr Cancer Netw. 2017:15(9):1131-9.

5. Hilal T, Gonzalez-Velez M, Prasad V. Limitations in clinical trials leading to anticancer drug approvals by the us food and drug administration. JAMA Intern Med. 2020;180(8):1108-15.

6. Kann BH, Johnson SB, Aerts HJWL, Mak RH, Nguyen PL. Changes in length and complexity of clinical practice guidelines in oncology, 1996-2019. JAMA Netw Open. 2020;3(3):e200841.

7. Akeson P, Larsson EM, Kristoffersen DT, Jonsson E, Holtås S. Brain metastases - comparison of gadodiamide injection-enhanced MR imaging at standard and high dose, contrast-enhanced CT and non-contrastenhanced MR imaging. Acta Radiol. 1995;36(3):300-6.

8. Schellinger PD, Meinck HM, Thron A. Diagnostic accuracy of MRI compared to CCT in patients with brain metastases. J Neuro-Oncol. 1999:44(3):275-81.

9. Ali A, Goffin JR, Arnold A, Ellis PM. Survival of patients with non-smallcell lung cancer after a diagnosis of brain metastases. Curr Oncol. 2013;20(4):e300-6.

10. Mystakidou K, Boviatsis EJ, Kouyialis AT, et al. Silent radiological imaging time in patients with brain metastasis. Clin Neurol Neurosurg. 2004;106(4):300-4.

11. Peters $S$, Camidge DR, Shaw AT, et al. Alectinib versus crizotinib in untreated alk-positive non-small-cell lung cancer. N Engl J Med. 2017;377(9):829-38.

12. Camidge DR, Kim HR, Ahn M-J, et al. Brigatinib versus crizotinib in alkpositive non-small-cell lung cancer. N Engl J Med. 2018;379(21):2027-39.

13. Nakagawa K, Garon EB, Seto T, et al. Ramucirumab plus erlotinib in patients with untreated, EGFR-mutated, advanced non-small-cell lung cancer (Relay): a randomised, double-blind, placebo-controlled, phase 3 trial. Lancet Oncol. 2019:20(12):1655-69.

14. West $\mathrm{H}, \mathrm{McCleod} \mathrm{M}$, Hussein $\mathrm{M}$, et al. Atezolizumab in combination with carboplatin plus nab-paclitaxel chemotherapy compared with chemotherapy alone as first-line treatment for metastatic non-squamous non-small-cell lung cancer (Impower130): a multicentre, randomised, open-label, phase 3 trial. Lancet Oncol. 2019;20(7):924-37.

15. Hellmann MD, Paz-Ares L, Bernabe Caro R, et al. Nivolumab plus ipilimumab in advanced non-small-cell lung cancer. N Engl J Med. 2019;381(21):2020-31.

16. Herbst RS, Giaccone G, de Marinis F, et al. Atezolizumab for firstline treatment of pd-I1-selected patients with nsclc. N Engl J Med. 2020;383(14):1328-39.

17. MokTSK, Wu Y-L, Kudaba I, et al. Pembrolizumab versus chemotherapy for previously untreated, PD-L1-expressing, locally advanced or metastatic non-small-cell lung cancer (KEYNOTE-042): a randomised, open-label, controlled, phase 3 trial. Lancet. 2019;393(10183):1819-30.

18. Paz-Ares L, Luft A, Vicente D, et al. Pembrolizumab plus chemotherapy for squamous non-small-cell lung cancer. N Engl J Med. 2018:379(21):2040-51

19. Wu Y-L, Cheng Y, Zhou $X$, et al. Dacomitinib versus gefitinib as first-line treatment for patients with EGFR-mutation-positive non-small-cell lung cancer (ARCHER 1050): a randomised, open-label, phase 3 trial. Lancet Oncol. 2017:18(11):1454-66.

20. Gandhi L, Rodríguez-Abreu D, Gadgeel S, et al. Pembrolizumab plus chemotherapy in metastatic non-small-cell lung cancer. N Engl J Med. 2018;378(22):2078-92
21. Soria J-C Ohe Y Vansteenkiste J, et al. Osimertinib in untreated egfr-mutated advanced non-small-cell lung cancer. N Engl J Med. 2018:378(2):113-25.

22. Soria J-C, Tan DSW, Chiari R, et al. First-line ceritinib versus platinumbased chemotherapy in advanced ALK-rearranged non-small-cell lung cancer (ASCEND-4): a randomised, open-label, phase 3 study. Lancet. 2017:389(10072):917-29.

23. Reck M, Rodríguez-Abreu D, Robinson AG, et al. Pembrolizumab versus chemotherapy for pd-I1-positive non-small-cell lung cancer. N Engl J Med. 2016;375(19):1823-33.

24. Thatcher N, Hirsch FR, Luft AV, et al. Necitumumab plus gemcitabine and cisplatin versus gemcitabine and cisplatin alone as first-line therapy in patients with stage IV squamous non-small-cell lung cancer (Squire): an open-label, randomised, controlled phase 3 trial. Lancet Oncol. 2015; 16(7):763-74.

\section{Publisher's Note}

Springer Nature remains neutral with regard to jurisdictional claims in published maps and institutional affiliations.
Ready to submit your research? Choose BMC and benefit from:

- fast, convenient online submission

- thorough peer review by experienced researchers in your field

- rapid publication on acceptance

- support for research data, including large and complex data types

- gold Open Access which fosters wider collaboration and increased citations

- maximum visibility for your research: over 100M website views per year

At BMC, research is always in progress.

Learn more biomedcentral.com/submissions 